\title{
Comparison of in-brace correction between supine, molded, asymmetric TLSOs and standing, CAD, symmetric TLSOs
}

\section{Speers}

\author{
From 9th International Conference on Conservative Management of Spinal Deformities - SOSORT 2012 \\ Annual Meeting \\ Milan, Italy. 10-12 May 2012
}

\section{Background}

Bracing for adolescent idiopathic scoliosis is a major component of conservative care. Many different brace designs exist, but they all must place corrective forces on the apex of the curves.

\section{Aim}

To determine whether asymmetric braces, molded supine, with manipulation during casting, compared to symmetric CAD designs, measured standing, offered better in brace correction.

\section{Methods}

34 females (ages 7 - 14) with adolescent idiopathic scoliosis were treated with a TLSO, and initial in brace radiographs were evaluated for correction of the curves. 22 received a symmetrical, posterior open CAD design made from standing measurements, and 12 received an asymmetric, posterior open design made from a supine casting with correction of the curve(s) applied during casting.

\section{Results}

For the 22 symmetric CAD TLSOs, initial in brace correction of thoracic curves was 9.1 degrees, with average out of brace thoracic curve 25.2 degrees, and lumbar curve correction of 10.3 degrees, with average out of brace lumbar curve 27.8 degrees. The percentage of correction in CAD versions was $36.1 \%$ thoracic, and $37.1 \%$ lumbar.

For the 12 asymmetric, TLSOs molded supine with manipulation, initial in brace correction of thoracic curves was 13.3 degrees, with average out of brace thoracic curve 26.7 degrees, and lumbar curve correction of 12.1 degrees, with average out of brace lumbar curve 26.6 degrees. The percentage of correction in supine, molded TLSOs was $49.8 \%$ thoracic, and $45.5 \%$ lumbar.

\section{Conclusion}

Supine, molded posterior open TLSOs with correction during casting provide greater initial in brace correction of both lumbar and thoracic curves compared to posterior open, symmetric, CAD designs made from standing measurements.

Published: 3 June 2013

\section{References}

1. Clin J, Aubin CE, Parent S, Labelle H: Biomechanical modeling of brace treatment of scoliosis: effects of gravitational loads. Med Biol Eng Comput 49(7):743-753.

2. Nachemson AL, Peterson LE: Effectiveness of treatment with a brace in girls who have adolescent idiopathic scoliosis. A prospective, controlled study based on data from the Brace Study of the Scoliosis Research Society. J Bone Joint Surg Am 1995, 77(6):815-822.

3. Rigo M, Reiter C, Weiss HR: Effect of conservative management on the prevalence of surgery in patients with adolescent idiopathic scoliosis. Pediatr Rehabil 2003, 6(3-4):209-214

doi:10.1186/1748-7161-8-S1-040

Cite this article as: Speers: Comparison of in-brace correction between supine, molded, asymmetric TLSOs and standing, CAD, symmetric TLSOs. Scoliosis 2013 8(Suppl 1):O40. 